REVIEW

\title{
Increased retinal blood flow in patients with Graves' disease: influence of thyroid function and ophthalmopathy
}

\author{
Yasuko Kurioka, Masaaki Inaba, Takahiko Kawagishi, Masanori Emoto, Yasuro Kumeda, Yuichi Inoue ${ }^{1}$, \\ Hirotoshi Morii and Yoshiki Nishizawa \\ Second Department of Internal Medicine and ${ }^{1}$ Department of Radiology, Osaka City University Medical School, 1-4-3, Asahi-machi, Abeno-ku, \\ Osaka 545, Japan \\ (Correspondence should be addressed to M Inaba, Second Department of Internal Medicine, Osaka City University Medical School, \\ 1-4-3, Asahi-machi, Abeno-ku, Osaka 545, Japan; Email: inaba-m@med.osaka-cu.ac.jp)
}

\begin{abstract}
Objective: Graves' ophthalmopathy (GO), resulting from the inflammation of retro-orbital tissue, is one of the major complications of Graves' disease (GD). We investigated the clinical usefulness of the measurement of retinal blood flow (RBF) in the evaluation of GO and its activity.

Measurement: RBF was quantitated by pulsed Doppler mode at just below the branch of central retinal artery, from which the resistance index (RI) was calculated.

Patients: Forty-seven euthyroid GD patients and 70 gender- and age-matched normal controls were measured for RI to investigate the effect of GO on RBF. To investigate the effect of hyperthyroidism, 20 GD patients were measured for RI changes during antithyroid drug (ATD) therapy. Furthermore, 17 GD patients with clinically overt GO were measured for RI changes during treatment with glucocorticoid plus retro-orbital radiation.

Results: RI and exophthalmos showed a significant positive correlation in 47 treated euthyroid GD patients without clinically overt GO $(r=0.307, P<0.05)$, but not in 70 age- and sex-matched normal subjects $(r=0.185, P=0.161)$. Furthermore, RI, but not exophthalmos, significantly correlated with serum TSH receptor antibodies, an indicator for the disease activity of GO. ATD therapy significantly reduced RI in GD patients from $0.719 \pm 0.041$ in the hyperthyroid state to $0.661 \pm 0.051$ in the euthyroid state, but not to the levels observed in normal subjects having the similar exophthalmos $(0.640 \pm 0.049)$. The fractional reduction of RI during ATD therapy significantly correlated with those of pulse pressure and ultrasonographic distensibility in carotid artery, but not with those of serum vascular injury markers. In 17 GD patients with clinically overt GO, all four patients having adipose tissue enlargement but not extraocular muscle hypertrophy (inactive GO) showed RI within the mean \pm 1 s.D. for treated GD patients without GO. In the other 13 GD patients having extraocular muscle hypertrophy (active GO), four and eight patients showed RI outside mean \pm 2 S.D. and mean \pm 1 S.D. respectively. Treatment with glucocorticoid plus radiation moved RI in 8 out of 10 patients toward the mean values of GD patients without GO, in spite of little improvement of exophthalmos.

Conclusions: It was suggested that GD patients showed altered retinal hemodynamics, possibly resulting either from the cardiovascular effect of hyperthyroidism or from retro-orbital inflammation, particularly in extraocular muscle.
\end{abstract}

European Journal of Endocrinology 144 99-107

\section{Introduction}

Graves' ophthalmopathy (GO), resulting from the inflammation of retro-orbital tissue, is the most common cause of exophthalmos (1-4). There is usually a close temporal relation between the onset of hyperthyroidism and GO (5), but GO can develop before and long after the onset of hyperthyroidism (6). Therefore, detecting its occurrence and activity in an early stage is important for its treatment (7). Although magnetic resonance imaging (MRI) is clinically useful for this purpose $(8,9)$, enormous medical costs discourage its use for patients with Graves' disease (GD).

The recent development of color Doppler ultrasound imaging of the ophthalmic vasculature allows easy examination of the pulsatile blood velocity in the central retinal artery and has therefore been used in the investigation of various vascular ophthalmic disorders $(10,11)$. The inflammatory changes in orbital fat, extraocular muscles and optic nerve in GO $(12,13)$, in addition to vascular endothelial injury 
observed in the hyperthyroid state (14), should induce hemodynamic alterations in retinal vessels (15). Furthermore, the distensibility of the walls of the carotid artery can be also determined as stiffness $\beta$ by echo-tracking sonography (16). A variety of serum vascular injury markers are elevated in GD patients (17-19), particularly in those having GO, suggesting vascular endothelial injury in retinal vessels in GD patients $(18,19)$. Furthermore, the cardiovascular effects of hyperthyroidism, characterized by an increase of cardiac output with a widened pulse pressure, should affect hemodynamic changes in retinal vessels (2) along with large vessels, such as the carotid artery.

This prompted us to determine the influence of exophthalmos and thyroid status on retinal blood flow (RBF). Furthermore, the correlation of the changes of RBF during antithyroid drug (ATD) therapy with those of hemodynamics and of serum vascular injury markers was examined to evaluate the clinical usefulness of RBF measurement for the detection of clinically active GO.

\section{Patients and methods}

\section{Patients}

GD patients $(n=84)$ and normal controls $(n=70)$ were enrolled in this study during a 20-month period from July 1996 to March 1998 after written informed consent was obtained from each patient. The diagnosis of GD was established by the presence of symptoms and signs of hyperthyroidism, a diffuse goiter, elevated serum levels of free thyroxine $\left(\mathrm{FT}_{4}\right)$ and free triiodothyronine $\left(\mathrm{FT}_{3}\right)$ with thyroid-stimulating hormone (TSH) levels below lower normal limit and with increased thyroid uptake of ${ }^{123}$ iodine before initiating ATD treatment. Thyroid radionuclide scans showed a diffuse pattern of radioiodine uptake. GD patients were divided into two major groups: those with and without clinically overt GO. Patients were considered to have clinically overt GO when one or more signs of infiltrative ophthalmopathy, including lid signs of retraction or lag, inflamed extraocular muscles, diplopia and chemosis were present on examination. GD patients with GO underwent MRI to discriminate between those with muscle enlargement and with excess adipose tissue. To determine the influence of exophthalmos on RBF separately from the hyperdynamic state resulting from hyperthyroidism, we studied 47 GD patients without clinically overt GO, who had been maintained in an euthyroid state for at least 3 months by taking $<5 \mathrm{mg}$ /day methimazole or $50 \mathrm{mg} /$ day propylthiouracil. As controls, $70 \mathrm{sex}-$ and age-matched normal subjects were enrolled. These controls were in euthyroid states and had negative tests for TSH receptor antibody (TRAb), thyroglobulin hemagglutination assay (TGHA) and microsome hemagglutination assay (MCHA).
To determine the influence of hyperthyroidism on RBF along with proptosis, 20 hyperthyroid GD patients without clinically overt GO were examined before and at least 3 months after normalization of thyroid function by ATD treatment.

GD patients with clinically overt GO $(n=17)$ were measured for their RBF along with proptosis before and after three cycles of intravenous pulse therapy each consisting of $3000 \mathrm{mg}$ of methylprednisolone and 10 doses of 2 Gy orbital irradiation over 2 weeks to a total of $20 \mathrm{~Gy}$.

To avoid confusion by other factors known to affect the retinal circulation, GD patients and normal controls with a history of orbital diseases were excluded. Furthermore: (i) hypertensive patients; (ii) patients taking medication known to affect hemodynamics; (iii) arteriosclerotic patients aged $\geq 60$ years or having atherosclerotic changes in the carotid arteries detected by high-resolution B-mode ultrasonography; (iv) smokers; (v) patients with major non-thyroidal illness; and (vi), patients having ocular disease that is known to affect RBF by raising intraocular pressure, such as primary retinal disease, vasculitis, vitreous disease, iritis, hyperopic patients, were excluded from this study for the same reason.

\section{Serum parameters}

Blood was drawn just before the ultrasound study after overnight fasting. $\mathrm{FT}_{3}, \mathrm{FT}_{4}$, and $\mathrm{TSH}$ levels were measured using commercially available kits (OrthoClinical Diagnostics, Amersham, UK) (21). TGHA and MCHA were also performed using commercially available kits (Fuji Rebio Co., Tokyo, Japan). TRAb was measured by a radioreceptor assay using a commercial kit (Baxter, Tokyo, Japan) (22). As serum vascular injury markers, serum levels of thrombomodulin, coagulation factor VIII, and von Willebrand factor were determined. Serum thrombomodulin was determined by a one-step sandwich enzyme immunoassay, the sensitivity of which is $1 \mu \mathrm{g} / \mathrm{l}$ for soluble thrombomodulin as previously described $(23,24)$. Coagulation factor VIII was measured using a chromogenic substrate method for photometric determination of prothrombin time as described previously (25). Von Willebrand factor was measured as described previously $(26,27)$.

\section{Exophthalmos}

Exophthalmos was measured by means of a Hertel's exophthalmometer (Handaya Co. Ltd, Tokyo, Japan) by the same examiner (M I) in all cases. Hertel exophthalmometer permits measurement of the distance between the lateral angle of the bony orbit and an imaginary perpendicular line tangent to the most anterior part of the cornea. 

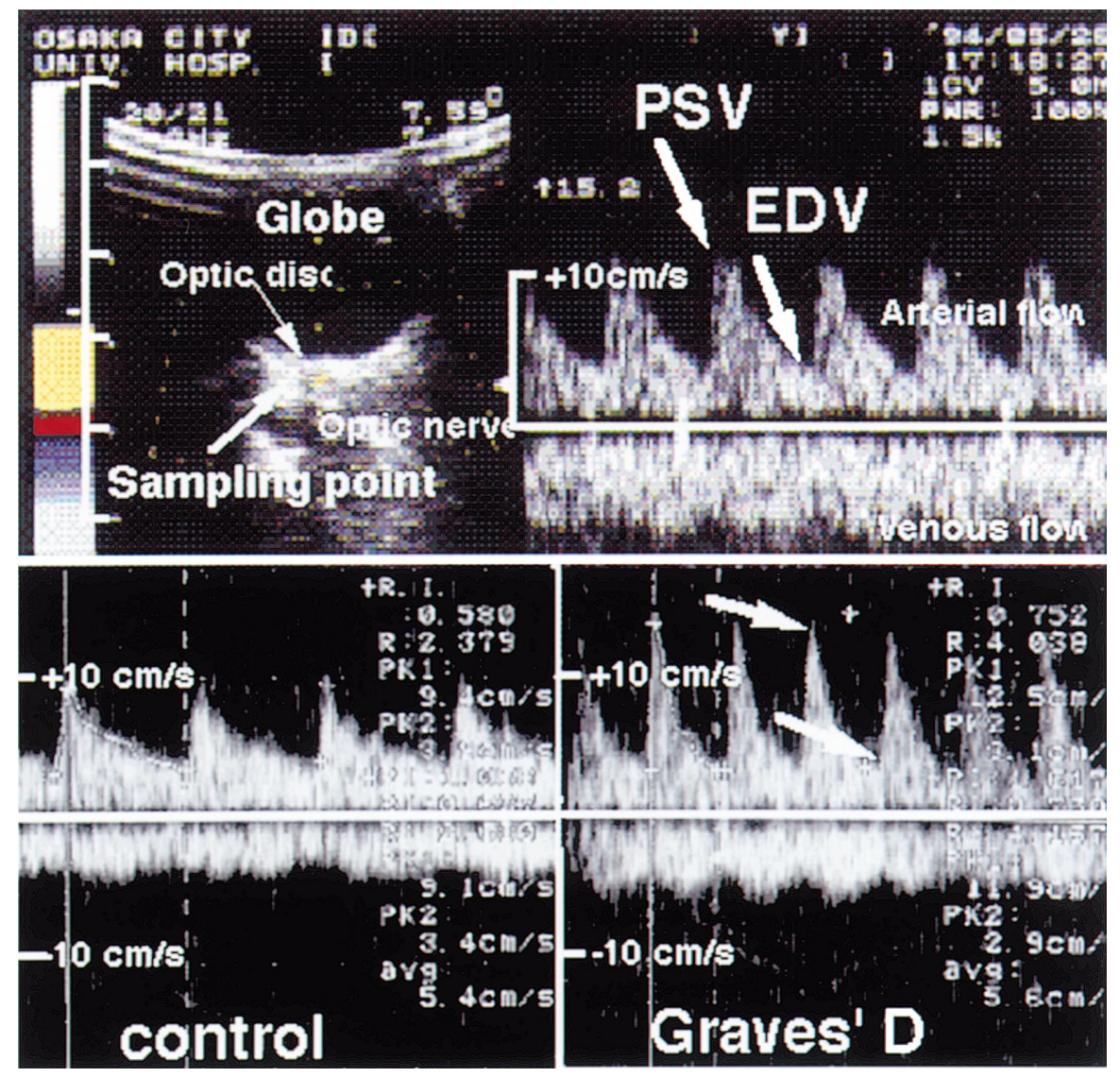

Figure 1 Duplex Doppler recording of central retinal arteries in patients with Graves' thyroid disease and normal controls. Upper panel: 'Doppler images' were used to determine the appropriate sampling point for pulsed Doppler recordings. A horizontal scan through the globe showed the central retinal artery within the anterior portion of the optic nerve shadow. The sample volume was positioned so that its center was about $3 \mathrm{~mm}$ behind the disc surface (arrow, upper left). The velocity waveform is displayed above the baseline to indicate the arterial blood flow (upper right). Lower panel: the PSV (arrow) was higher in Graves' patients (right lower panel) than in the control subjects (left lower panel).

\section{Duplex Doppler sonography}

Ultrasound examinations were performed in left eyes as reported previously (10). Briefly, images were obtained with a duplex Doppler apparatus (Aloka SSD 2000, Aloka, Tokyo, Japan) with a $5 \mathrm{MHz}$ convex array probe in both the color Doppler and pulsed Doppler mode. The peak systolic flow velocity (PSV), the end-diastolic flow velocity (EDV), and the time-averaged flow velocity (TAV) were automatically calculated by the ultrasound apparatus (Fig. 1). The resistance index (RI), a measure of the peripheral resistance to flow in arteries $(11,28)$, was determined as follows: $\mathrm{RI}=(\mathrm{PSV}-\mathrm{EDV}) / \mathrm{PSV}$. All measurements were performed by the same examiner (Y K) who was unaware of subject characteristics. Because coefficient of the between day variation of RI determined from ten normal controls was only $2.57 \pm$ $2.14 \%$, the RI value was highly reproducible and thus reliable, as previously reported $(10,11)$.

\section{Stiffness $\beta$ (ultrasonographic measurements of arterial distensibility)}

Vessel diameter and pulsatile diameter changes were measured by means of echo-tracking sonography (10, 28-30) by the same examiner (Y K). We used a recently developed ultrasound echo-tracking instrument interfaced with a real-time ultrasound scanner and fitted with a 3.5- and 5-MHz linear array transducer (Aloka SSD 610, Aloka Co. Ltd, Tokyo, Japan) that is capable of detecting vessel wall movements of $<10 \mu \mathrm{m}$ at the level of bifurcation in the common carotid artery $(16,31,32)$. This technique has recently been used to evaluate stiffness in the common carotid artery and the abdominal aorta in healthy men and women of different ages (33-35). Details of the study technique have been described previously $(36,37)$. The distensibility of the arterial walls was expressed as stiffness $\beta$ (16), which was 
Table 1 Clinical profiles of patients with Graves' diseases and normal controls.

\begin{tabular}{|c|c|c|c|}
\hline & Graves' disease $(n=47)$ & Normal $(n=70)$ & $P$ value \\
\hline Female/male & $45 / 2$ & $64 / 6$ & $>0.05$ \\
\hline Age (year) & $36.5 \pm 13.1$ & $35.8 \pm 14.3$ & $>0.05$ \\
\hline Systolic BP (mmHg) & $115.8 \pm 11.8$ & $121.0 \pm 18.3$ & $>0.05$ \\
\hline Diastolic BP (mmHg) & $70.5 \pm 7.5$ & $75.5 \pm 9.0$ & $>0.05$ \\
\hline Mean BP $(\mathrm{mmHg})$ & $85.6 \pm 7.4$ & $88.5 \pm 12.3$ & $>0.05$ \\
\hline Pulse pressure $(\mathrm{mmHg})$ & $45.3 \pm 11.4$ & $48.2 \pm 10.9$ & $>0.05$ \\
\hline F-T3 $(\mathrm{pg} / \mathrm{ml})$ & $3.87 \pm 1.54$ & $3.49 \pm 0.98$ & $>0.05$ \\
\hline F-T4 (ng/dl) & $1.40 \pm 1.12$ & $1.38 \pm 1.11$ & $>0.05$ \\
\hline TSH (mlU/ml) & $1.60 \pm 4.15$ & $2.19 \pm 1.22$ & $>0.05$ \\
\hline $\operatorname{TRAb}(\%)$ & $25.8 \pm 24.9$ & N.D. & \\
\hline Right proptosis (mm) & $17.0 \pm 2.7$ & $14.3 \pm 3.1$ & $<0.0001^{*}$ \\
\hline Left proptosis (mm) & $17.0 \pm 2.8$ & $14.5 \pm 2.6$ & $<0.0001^{*}$ \\
\hline Right resistance index & $0.668 \pm 0.051$ & $0.642 \pm 0.052$ & $0.001^{*}$ \\
\hline Left resistance index & $0.665 \pm 0.053$ & $0.640 \pm 0.049$ & $0.005^{\star}$ \\
\hline
\end{tabular}

calculated as follows: stiffness $\beta=[\operatorname{In}(\mathrm{Ps} / \mathrm{Pd})] \times$ $\mathrm{Dd} /(\mathrm{Ds}-\mathrm{Dd})$, where Ps and Pd denote the maximal systolic and end-diastolic blood pressures, expressed in $\mathrm{mmHg}$ respectively. Ds and Dd are the systolic and diastolic inner diameters $(\mathrm{mm})$ of the artery, respectively. Each subject was examined three times in the right common carotid artery with calculation of stiffness from the corresponding diameter, pulsatile diameter changes, and blood pressures obtained by the auscultatory method, as previously reported (29).

\section{MRI}

MRI with a $1.5 \mathrm{~T}$ superconductive magnetic unit (SMT$150 X)$ was performed in the 20 untreated GD patients as previously reported (8). The pulse sequence was T1weighted spin echo (500-650/20 ms), and the technical parameters for imaging included two excitations, a $15 \mathrm{~cm}$ field of view, a $192 \times 256$ matrix and a $5 \mathrm{~mm}$ section thickness. Muscle changes were evaluated by using coronal section at $90^{\circ}$ from the right or left optic nerve. The degree of muscle swelling was expressed as a ratio of the maximal muscle thickness to the diameter of the ipsilateral optic nerve to minimize the interindividual difference in muscle thickness (normal ratio $<1.0)$, as previously described (8).

\section{Statistical analysis}

Data are expressed as means \pm S.D. unless otherwise indicated. Statistical analyses of RI values between GD patients without GO and normal controls were performed by using one-way analysis of variance (ANOVA) and multiple comparison (Scheffe's F type) for the assessment of mean. The changes of RI values by ATD treatment were compared with the two-tailed Student's $t$-test for paired data.

\section{Results}

\section{RI values in the central retinal artery in euthyroid GD patients and normal controls and its correlation with exophthalmos and serum TRAb}

As shown in Table 1, the difference in RI in the central retinal artery was measured in 47 GD patients (M/F, 2/ 45) maintained in a euthyroid state by ATD treatment and the 70 normal controls (M/F, 6/64). GD patients and normal subjects were matched by gender, age, and blood pressure. Furthermore, there was no significant difference in serum $\mathrm{FT}_{3}, \mathrm{FT}_{4}$, or TSH between GD patients and normal subjects. Mean values of proptosis were significantly greater in euthyroid GD patients $(17.0 \pm 2.8 \mathrm{~mm})$ than in normal subjects $(14.8 \pm$ $2.2 \mathrm{~mm}, P<0.0001)$, consistent with the reported values of $13.9 \pm 1.9 \mathrm{~mm}$ in 538 normal Japanese (38). RI values were significantly greater in euthyroid GD patients without GO than in normal controls. Furthermore, as shown in Fig. 2, RI values were correlated significantly in a positive manner with proptosis in these GD patients $(r=0.307, P<0.05)$, but not in normal subjects $(r=0.185, P=0.161)$. The solid and dotted lines represent the mean, mean \pm s.D. and mean \pm 2 S.D. of RI values respectively, on the basis of their proptosis in these 47 GD patients without GO. All of these 47 patients showed RI values within the 

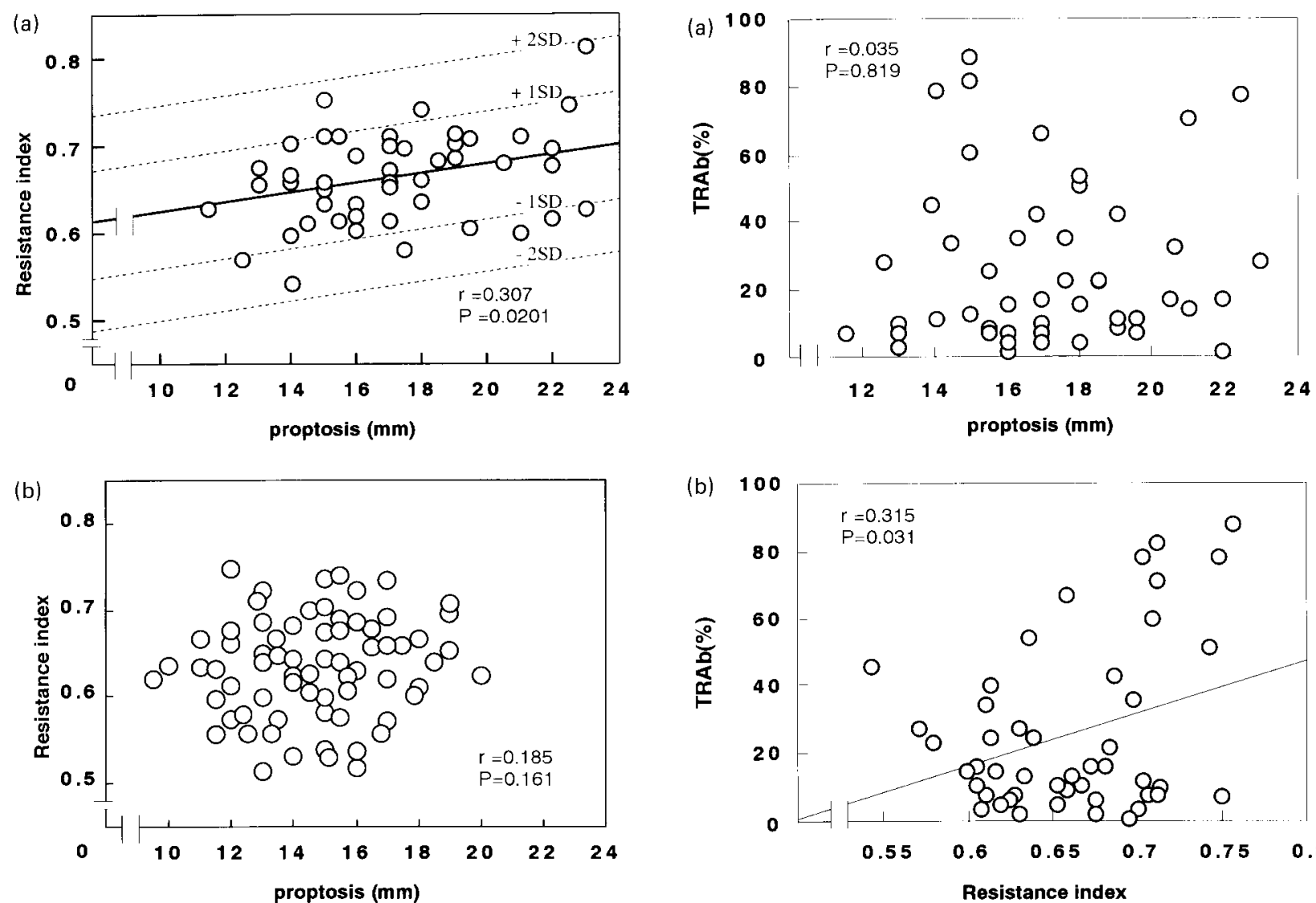

Figure 2 Relationship between RI values in the central retinal artery and proptosis. The correlation between the two parameters was examined in antithyroid drug-treated euthyroid GD patients without clinically overt GO who had been maintained in a euthyroid state for at least 3 months by taking $<5 \mathrm{mg} /$ day methimazole or $50 \mathrm{mg} /$ day propylthiouracil (upper panel) and in euthyroid normal controls (lower panel). A significant positive correlation was found between $\mathrm{RI}$ values and proptosis in euthyroid Graves' patients $(r=0.307, P=0.020, n=47)$, but not in normal controls $(r=0.185, P=0.161, n=70)$. All $47 \mathrm{GD}$ patients show $\mathrm{RI}$ values inside the mean \pm 2 S.D. on the basis of their proptosis.

mean \pm 2 s.D., and 39 of 47 fell within the mean \pm S.D. on the basis of their proptosis.

As shown in Fig. 3, serum TRAb, which is known as a good indicator for the disease activity of GO (39), was correlated significantly in a positive manner with RI values but not with exophtalmos.

\section{Dependence of RI values in the central retinal artery on thyroid function}

As shown in Fig. 4, RI values in $20 \mathrm{GD}$ patients decreased significantly from $0.719 \pm 0.041$ in the hyperthyroid state to $0.661 \pm 0.051$ in the euthyroid state during ATD treatment, but not to the levels $(0.642 \pm 0.052)$ observed in the normal subjects having the similar proptosis obtained from the data in

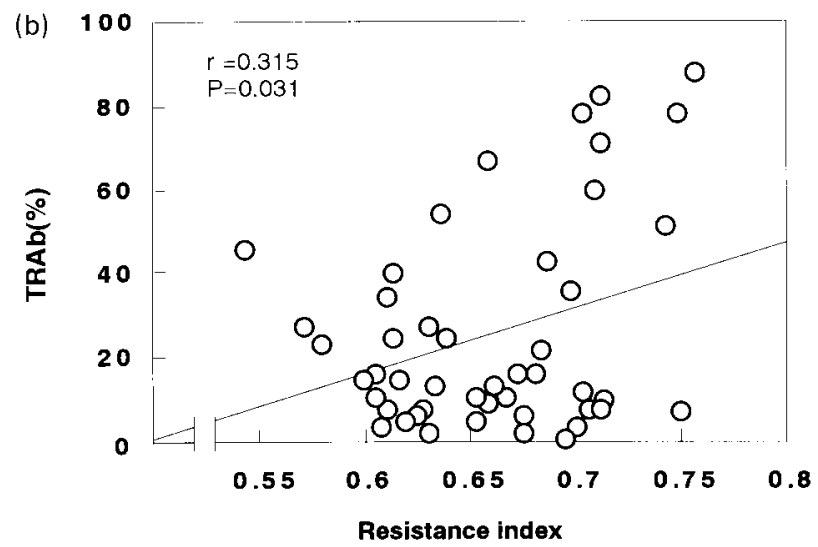

Figure 3 Relationship between serum TRAb and proptosis or RI values in the central retinal artery. The correlation of serum TRAb with proptosis (upper panel) and with RI values in the central retinal artery (lower panel) was examined in antithyroid drug-treated euthyroid GD patients without clinically overt GO who had been maintained in a euthyroid state for at least 3 months by taking $<5 \mathrm{mg} /$ day methimazole or $50 \mathrm{mg} /$ day propylthiouracil. Serum TRAb levels were significantly correlated in a positive manner with $\mathrm{RI}$ values $(r=0.315, P=0.031$, $n=47)$, but not with proptosis $(r=0.035, P=0.819, n=47)$.

Table 1. By contrast, the mean proptosis values for the $20 \mathrm{GD}$ patients were essentially unchanged from $16.1 \pm 2.4 \mathrm{~mm}$ to $16.0 \pm 2.3 \mathrm{~mm}$ after ATD treatment. The fractional reduction of RI values by ATD treatment was significantly correlated with that of stiffness $\beta$ in the right carotid artery or that of pulse pressure, but not with that of proptosis, $\mathrm{FT}_{3}$ or $\mathrm{FT}_{4}$ concentration (Table 2).

\section{Correlation of RI values in the central retinal artery with serum markers for vascular injury}

Serum von Willebrand factor and factor VIII reduced significantly $(P<0.0001)$ and levels of thrombomodulin tended to reduce, although not significantly, with normalization of thyroid function (data not 


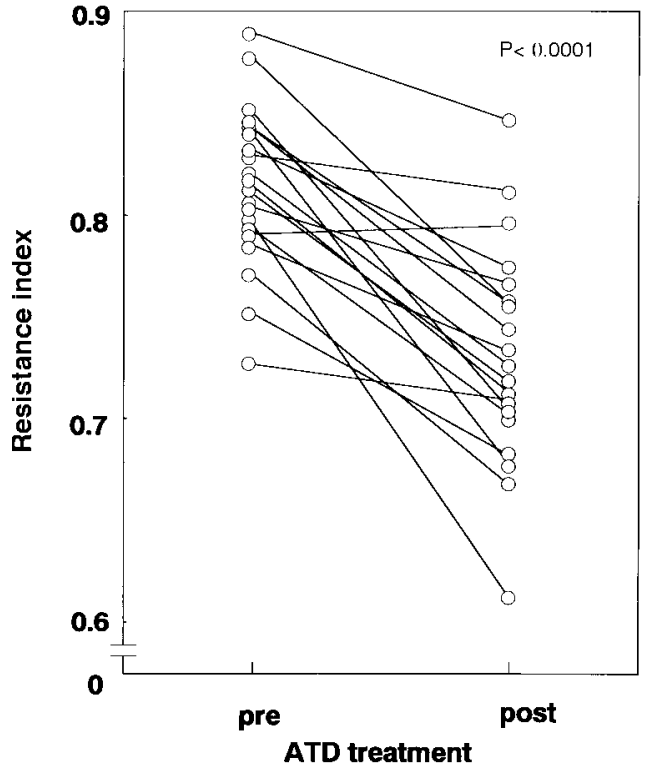

Figure 4 Changes in RI values in GD patients without clinically evident GO during normalization of thyroid function by antithyroid drug treatment. $\mathrm{RI}$ values in $20 \mathrm{GD}$ patients decreased significantly from $0.719 \pm 0.041$ to $0.661 \pm 0.051(P<0.0001)$ during treatment with antithyroid drugs except 1 patient, in spite of the lack of apparent change in proptosis from $16.1 \pm 2.4$ to $16.0 \pm 2.3 \mathrm{~mm}$

shown). The fractional reductions of these serum vascular injury markers during ATD treatment were not significantly correlated with that of RI values (Table 2 ). Serum levels of any vascular injury markers did not differ significantly between patients with and without clinically overt GO, either in a hyperthyroid or an euthyroid state (data not shown).

Table 2 Relationship between changes in RI values and other parameters during ATD treatment.

\begin{tabular}{lcc}
\hline$\Delta$ RI/RI before & $\boldsymbol{r}$ value & $\boldsymbol{P}$ value \\
\hline$\Delta$ proptosis/proptosis before & 0.268 & 0.2166 \\
Hemodynamic parameters & & \\
$\Delta$ stiffness $\beta /$ stiffness $\beta$ before & 0.387 & $0.0479^{*}$ \\
$\Delta$ pulse pressure/pulse pressure before & 0.446 & $0.0331^{*}$ \\
$\Delta$ mean BP/mean BP before & 0.215 & 0.3244 \\
Serum parameters & 0.169 & 0.4509 \\
$\Delta$ VIII factor/VIII factor before & 0.037 & 0.8706 \\
$\Delta$ thrombomodulin/thrombomodulin before & 0.063 & 0.7819 \\
$\Delta$ von Willebrand factor/von Willebrand & & \\
$\quad$ factor before & 0.029 & 0.8949 \\
$\Delta$ FT3/FT3 before & 0.009 & 0.9670 \\
$\Delta$ FT4/FT4 before & 0.039 & 0.8779 \\
$\Delta$ TSH/TSH before &
\end{tabular}

* Statistically significant $(P<0.05)$ by one-way ANOVA with Scheffe's $\mathrm{F}$ test.

The fractional reduction $(\Delta)$ was calculated by dividing the changes during ATD treatment by pretreatment value.

\section{RI values in the central retinal artery in GD patients with clinically overt GO after treatment with glucocorticoid and orbital irradiation}

Figure 5 shows the relationship between RI and exophthalmos in GD patients with overt GO and the effect of glucocorticoid and orbital irradiation. The solid line and dotted lines represent the mean, mean \pm 1 s.D. and mean \pm 2 s.D. for RI values in the 47 treated euthyroid GD patients without GO (also as shown in Fig. 2). GD patients with overt GO with a ratio of the maximal muscle thickness to the diameter of the ipsilateral optic nerve above 1.9 and less than 1.0 were regarded as having extraocular muscle hypertrophy and adipose tissue enlargement respectively (8). Among the 17 GD patients with overt GO, all four patients having signs of adipose tissue enlargement but not extraocular hypertrophy on MRI showed RI values within the mean \pm s.D. Among the other $13 \mathrm{GD}$ patients showing extraocular muscle hypertrophy on MRI, 4, 4, and 5 patients showed RI values falling outside the mean \pm 2 s.D., between mean \pm 2 s.D. and mean \pm s.D., and within the mean \pm S.D. respectively. Glucocorticoid and radiotherapy moved RI values in 8 out of 10 patients towards the regression line of RI values of treated GD patients without GO in spite of little improvement of proptosis from $19.6 \pm 4.6 \mathrm{~mm}$ to $18.9 \pm 4.0 \mathrm{~mm}$ during the treatment.

\section{Discussion}

The main cardiovascular hemodynamic changes in hyperthyroidism include reductions in systemic vascular resistance and diastolic blood pressure, and increases in heart rate, cardiac contractility, cardiac output, systolic blood pressure, diastolic relaxation, and myocardial oxygen consumption $(15,20)$. As a result, cardiac output increases and pulse pressure widens. The increase of RI values in hyperthyroid GD patients was attenuated as thyroid function was normalized by ATD treatment. Furthermore, the fractional reduction of RI values during ATD treatment significantly correlated with those of pulse pressure and of stiffness $\beta$ in the carotid artery (Table 2). Therefore, it was strongly suggested that increased peripheral vascular resistance of the central retinal artery, as reflected by an increase in RI value, was caused by overperfusion resulting from increased cardiac output with widened pulse pressure in hyperthyroid state, thus rationalizing the use of RI values as an index of RBF. However, RI values remained significantly elevated in GD patients even 3 months after thyroid function was normalized, suggesting the presence of another mechanism by which RI values were increased. A strong candidate mechanism is inflammation of retro-orbital tissue by autoimmune processes, which are known to be associated with GO. Because retro-orbital inflammation 


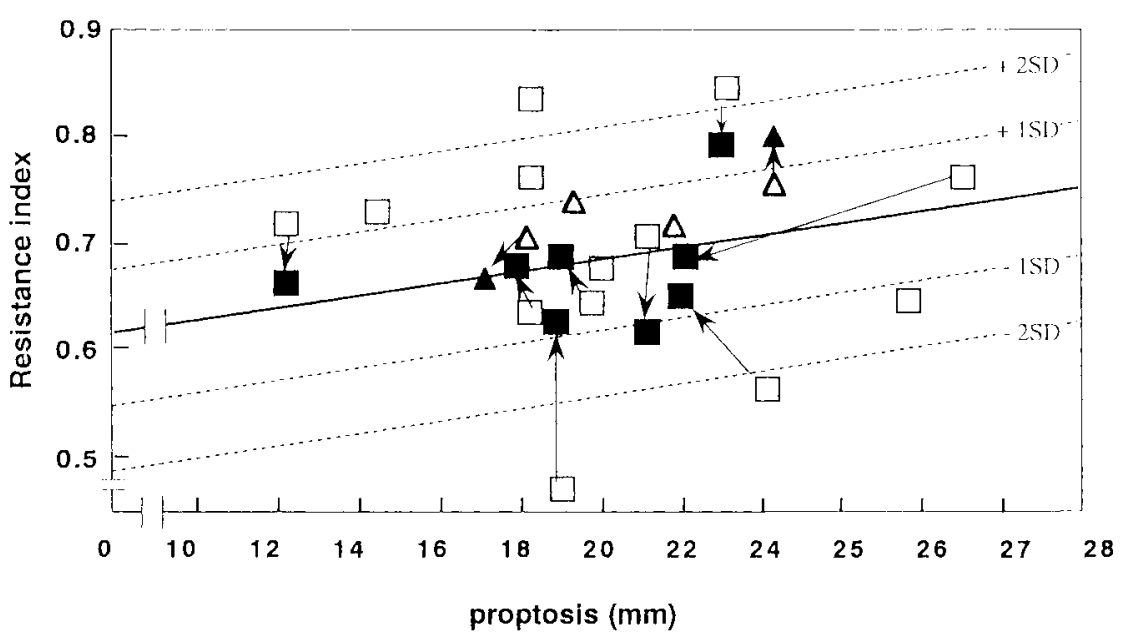

Figure 5 Individual changes in RI values in comparison with proptosis during treatment with glucocorticoid plus retro-orbital irradiation in GD patients with clinically overt GO. Open and closed rectangles indicate the patients showing extraocular muscle swelling documented by MRI before and after treatment respectively. Open and closed triangles indicate the patients with adipose tissue enlargement documented by MRI before and after treatment respectively. The solid and dotted lines represent the mean, mean \pm 1 S.D. and mean \pm 2 S.D. for euthyroid GD patients without GO, as shown in Fig. 2. The proportions of GD patients showing RI values for proptosis outside the mean \pm 1 S.D., and the mean \pm 2 S.D., were significantly higher in the patients with clinically overt GO $(47.1 \%$ (8 of 17 patients) and $23.5 \%$ (4 of 17 patients) respectively) than in the patients without GO (17.0\% (8 of 47 patients) and $0 \%$ (0 of 47 patients) respectively). Among GD patients with GO, all four patients having adipose tissue enlargement showed RI values falling within mean \pm 1 S.D. RI values in 4 and 8 patients out of the other $13 \mathrm{GD}$ patients having extraocular muscle hypertrophy fell outside mean \pm 2 S.D. and mean \pm 1 S.D. respectively. Treatment with glucocorticoid plus retro-orbital irradiation moved the RI values of GD patients with overt GO in 8 out of 10 patients towards the mean values for GD patients without GO.

can cause overperfusion of the central retinal artery as in other tissues (39), it might cause an increase of RI values along with proptosis. These factors may explain the positive correlation between proptosis and RI values in GD patients and the lack of such a correlation in normal subjects. Furthermore, the contribution of retro-orbital inflammation to increased RI values was supported by a significant positive correlation between RI values and serum TRAb, a good indicator for the disease activity of GO (39). Of 17 GD patients with clinically overt GO, two patients showed RI values for their proptosis greater than the mean +2 s.D., whereas two patients had values lower than the mean -2 s.D. (Fig. 4). Because we excluded patients with the other ocular diseases that might affect measurement of RBF, it is possible that the abnormally high RI values may be related to the presence of severe autoimmune congestive inflammatory orbitopathy. This notion is supported by the data that, when GD patients developed clinically overt GO resulting from adipose tissue enlargement but not extraocular muscle swelling (inactive retro-orbital inflammation), they did not show any increase in RI values (Fig. 5). The abnormally low RI values in some patients could be explained by compression of the central retinal artery often observed in GD patients with GO $(40,41)$. Steroid and radiotherapy improved extraocular muscle swelling in clinically overt GO on MRI, an indicator for the disease activity of GO, in spite of little improvement of proptosis, an indicator for the disease severity of GO. These treatments moved RI values of those patients toward the mean values for GD patients without GO. Together with a significant positive correlation with serum TRAb, RI values might be useful for the assessment of the disease activity of GO.

Next, in order to evaluate the relationship between RI values and vascular injury, we measured serum markers for vascular injury, including thrombomodulin, von Willebrand factor, and factor VIII, as serum levels of these markers are reported to be higher in GD patients with GO than in those without (42-47). Each marker reflects vascular injury at different sites of endothelial damage. In contrast to the previous reports, none of markers were significantly different between GD patients with and without clinically overt GO either in a hyperthyorid or an euthyroid state. Furthermore, the fractional reductions of serum vascular injury markers during ATD treatment did not correlate significantly with those of RI values, stiffness $\beta$ or proptosis. Therefore, measurement of serum concentrations of vascular injury markers such as thrombomodulin, von Willebrand factor, or factor VIII, is unlikely to be clinically useful in evaluating GO.

In summary, we have shown that waveform analysis of RBF using duplex Doppler sonography to be a reliable noninvasive technique and that it detected hemodynamic changes in GD patients even without GO. The presence of active GO may specifically contribute to increased RBF, because of: (i) a selective increase of RI values in GD patients having extraocular muscle 
swelling, but not adipose tissue enlargement; and (ii), a significant reduction in RI values in spite of little improvement in proptosis by glucocorticoid plus retroorbital irradiation. Our findings raise the possibility that this technique represents a clinically useful adjunct to MRI for detection and follow up of GO after normalization of thyroid function in GD patients and possibly for the determination of its activity.

\section{References}

1 Mulvaney JH. Exophthalmos of hyperthyroidism: A differentiation in mechanism, pathology, symptomatology and treatment of two varieties. American Journal of Ophthalmology 194427 589693,820

2 Brain R. Pathogenesis and treatment of endocrine exophthalmos. Lancet 1959 i $109-115$.

3 Weetman A. Thyroid-associated eye disease: pathophysiology. Lancet $199133825-28$.

4 Bahn RS \& Heufelder AE. Pathogenesis of Graves' ophthalmopathy. New England Journal of Medicine 199320 1468-1475.

5 Bartley GB \& Fatourechi V. The chronology of Graves' ophthalmopathy after treatment for Graves's hyperthyroidism. New England Journal of Medicine 1992326 1733-1738.

6 Gorman CA. Temporal relationship between onset of Graves' ophthalmopathy and diagnosis of thyrotoxicosis. Mayo Clinic Proceedings 198358 515-519.

7 Villadolid MC, Yokoyama N, Izumi M, Nishikawa T, Kimura H, Ashizawa $\mathrm{K}$ et al. Untreated Graves' disease patients without clinical ophthalmopathy demonstrate a high frequency of extraocular muscle (EOM) enlargement by magnetic resonance. Journal of Clinical Endocrinology and Metabolism 199580 28302833.

8 Nishikawa M, Yoshimura M, Toyoda N, Masaki N, Yonemoto T, Gondou A et al. Correlation of orbital muscle changes evaluated by magnetic resonance imaging and thyroid-stimulating antibody in patients with Graves' ophthalmopathy. Acta Endocrinology $1993129213-219$.

9 Utech CI, Khatibnia U, Winter PF \& Wulle KG. MR T2 relaxation time for the assessment of retrobulbar inflammation in Graves' ophthalmopathy. Thyroid 19955 185-193.

10 Kawagishi T, Nishizawa Y, Emoto M, Konishi T, Maekawa K, Hagiwara $S$ et al. Impaired retinal artery blood flow in IDDM patients before clinical manifestations of diabetic retinopathy. Diabetes Care 199518 1544-1549.

11 Lieb WE, Cohen SM, Merton DA, Shields JA, Mitchell DG \& Goldberg BB. Color Doppler imaging of the eye and orbit. Archives of Ophthalmology 1991109 527-531.

12 Coleman DJ, Jack RL, Franzen LA \& Werner SC. High resolution B scan ultrasonography of the orbit. Archives of Ophthalmology 197288 465-471.

13 Naffziger HC. Pathologic changes in the orbit in progressive exophthalmos with special reference to alterations in extraocular muscles and optic disk. Archives of Ophthalmology 19339 $1-12$.

14 Parker JLW \& Lawson DH. Death from thyrotoxicosis. Lancet 1973 ii $894-895$.

15 Klein I. Thyroid hormone and the cardiovascular system. American Journal of Medicine $1990 \mathbf{8 8} 631-637$.

16 Kawasaki T, Sasayama S, Yagi S-I, Asakawa T \& Hirai T. Noninvasive assessment of the age related changes in stiffness of major branches of the human arteries. Cardiovascular Research $198721678-687$.

17 Simone JV, Abildgaard CF \& Schulman I. Blood coagulation in thyroid function. New England Journal of Medicine 1965273 1057-1061.

18 Morikawa Y, Morikawa A \& Makino I. Relationship of thyroid states and serum thrombomodulin (TM) levels in patients with
Graves' disease: TM, a possible new marker of the peripheral activity of thyroid hormones. Journal of Clinical Endocrinology and Metabolism 199376 609-614.

19 Takano S, Kimura N, Ohdama S \& Aoki N. Plasma thrombomodulin in health and diseases. Blood Vessels 199076 2024-2029.

20 Saito I, Ito K \& Saruta T. The effect of age on blood pressure in hyperthyroidism. Journal of the American Geriatric Society 1985 33 19-22.

21 Christofides ND \& Sheehan CD. Enhanced chemiluminescence labeled-antibody immunoassay (Amerlite-MAB) for free thyroxine: design, development, and technical validation. Clinical Chemistry 199541 17-23.

22 Shewring G \& Smith BR. An improved radioreceptor assay for TSH receptor antibodies. Clinical Endocrinology 1982 17 409-417.

23 Kodama S, Uchijima E, Nagai M, Mikawatani K, Hayashi T \& Suzuki K. One-step enzyme immunoassay for soluble human thrombomodulin using monoclonal antibodies. Clinica Chimica Acta 1990192 191-200.

24 Ishii H, Uchiyama H \& Kazama M. Soluble thrombomodulin antigen in conditioned medium is increased by damage of endothelial cells. Thrombosis and Haemostasis 199165 618-623.

25 Dati F, Barthels M, Conrad J, Fluckiger J, Girolami A, Hanseler E et al. Multicenter evaluation of a chromogenic substrate method for photometric determination of prothrombin time. Thrombosis and Haemostasis $1987 \mathbf{2 8} 856-865$.

26 Norris CS \& Barnes RW. Renal artery flow velocity analysis: a sensitive measure of experimental and clinical renovascular resistance. Journal of Surgical Research 198436 230-236.

27 Macfarlane DE, Stibbe J, Kirby EP, Zucker MB, Grant RA \& McPherson J. A method for assaying von Willebrand factor (Ristocetin cofactor). Thrombosis et Diathesis Haemorrhagica 1975 34 306-308.

28 Emoto M, Nishizawa Y, Kawagishi T, Maekawa K, Hiura Y Kanda $\mathrm{H}$ et al. Stiffness Indexes of the common carotid and femoral arteries are associated with insulin resistance NIDDM. Diabetes Care 199821 1178-1182.

29 Lanne T, Soneson B, Bergqvist D, Bengtsson H \& Gustafsson D. Diameter and compliance in the male human abdominal aorta: influence of age and aortic aneurysm. European Journal of Vascular Surgery 19926 178-184.

30 Hokansson DE, Mozersky DJ, Sumner DJ \& Strandness DE Jr. A phase-locked echo-tracking system for recording arterial diameter changes in vivo. Journal of Applied Physiology 197232 $728-733$.

31 Lindstom K, Gennser G, Sindberg-Eriksson P, Bentin M \& Dahl P. An improved echo-tracker for studies on pulse waves in the fetal aorta. Ed. P Rolfe. In Fetal Physiological Measurements, pp 217226. London: Butterworths, 1978

32 Benthin M, Dahl P, Ruzicka R \& Lindstrom K. Calculation of pulse wave velocity using cross correlations effects of reflexes in the arterial tree. Ultrasound Medical Biology 199117 461-469.

33 Sonesson B, Hansen F, Stale H \& Lanne T. Compliance and diameter in the human abdominal aorta-the influence of age and sex. European Journal of Vascular Surgery 19937 690-697.

34 Hansen F, Mangell P, Sonesson B \& Lanne T. Diameter and compliance in the human common carotid artery-variations with age and sex. Ultrasound Medical Biology 199521 1-9.

35 Arndt JO, Klauske J \& Mersch F. The diameter of the intact carotid artery in man and its change with pulse pressure. Pflugers Archives 1968301 230-240.

36 Lanne T, Stale H \& Bengtsson H. Noninvasive measurement of diameter changes in the distal abdominal aorta in man. Ultrasound Medical Biology 199218 451-457.

37 Hansen F, Bergqvist D, Mangell P, Ryden A, Sonesson B \& Lanne T. Non-invasive measurement of pulsatile vessel diameter changes and elastic properties in human arteries: a methodological study. Clinical Physiology 199313 631-643.

38 Amino N, Yuasa T, Yabu Y, Miyai K \& Kumahara Y. Exophthalmos in autoimmune thyroid disease. Journal of Clinical Endocrinology and Metabolism 198051 1232-1234. 
39 Gerding MN, van der Meer JWC, Broenink M, Bakker O, Wiersinga WM \& Prummel MF. Association of thyrtrophin receptor antibodies with the clinical features of Graves' ophthalmopathy. Clinical Endocrinology 200052 267-271.

40 Walsh KM, Leen E, MacSween RN \& Morris AJ. Hepatic blood flow changes in chronic hepatitis C measured by duplex Doppler color sonography: relationship to histological features. Digestive Diseases and Sciences $1998432584-2590$.

41 Neigel JM, Rootman J \& Belken RI. Dysthyroid optic neuropathy. Ophthalmology 198895 1515-1521.

42 Kazama M. Clinical evaluation of hemostatic molecular markers. Acta Haematologica Japonica 198851 1387-1394.

43 Trobe JD. Optic nerve involvement in dysthyroidism. Ophthalmology $1981 \mathbf{8 8} 488-492$.

44 Rogers GS, Shane SR \& Jencks FS. Factor VIII activity and thyroid function. Annals of Internal Medicine 198297 713-716.
45 Uchiyama H, Hiraishi S, Ohtani H, Ishii H \& Kazama M. Soluble thrombomodulin antigen in conditioned medium is increased by damaged of endothelial cells. Thrombosis and Haemostasis 1991 65 618-623.

46 Giddings JC, Coles P \& Williams BD. Comparison of thrombomodulin and von Willebrand factor antigen in human plasma in various diseases. Thrombosis and Haemostasis 198962333.

47 Blann AD \& NcCollum CN. Von Willebrand factor, endothelial cell damage and atherosclerosis. European Journal of Vascular Surgery 19948 10-15.

Received 12 April 2000

Accepted 31 August 2000 